\title{
Evaluación de la toxicidad vaginal de Croton lechleri en conejas
}

\author{
Assessment of Croton lechleri vaginal toxicity in rabbits
}

\author{
Salomón Ayala , Juan Rojas², David Díaz³, José Juárez4, Carlos Delgado² \\ ${ }^{1}$ Centro de Investigación de Bioquímica y Nutrición, Facultad de Medicina, Universidad Nacional Mayor de San Marcos. Lima, Perú. \\ ${ }^{2}$ Instituto de Investigaciones Clínicas, Facultad de Medicina, Universidad Nacional Mayor de San Marcos. Lima, Perú. \\ ${ }^{3}$ Instituto de Patología, Facultad de Medicina, Universidad Nacional Mayor de San Marcos. Lima, Perú. \\ ${ }^{4}$ Facultad de Farmacia y Bioquímica, Universidad Nacional Mayor de San Marcos. Lima, Perú.
}

\begin{abstract}
Resumen
Objetivo: Evaluar la toxicidad vaginal de una preparación en gel de Croton lechleri en conejas. Diseño: Estudio experimental. Institución: Centro de Investigación de Bioquímica y Nutrición Alberto Guzmán Barrón, Instituto de Investigaciones Clínicas, Instituto de Patología de la Facultad de Medicina, y Facultad de Farmacia, Universidad Nacional Mayor de San Marcos, Lima, Perú. Material biológico: Resina de Croton lechleri y conejos hembras, blancos, Nueva Zelanda. Métodos: Los animales fueron distribuidos en tres grupos ( $n=5)$ : I. Control, vehículo; II, C. lechleri 1\%; y III, C. lechleri $5 \%$; los conejos recibieron estas muestras en un volumen de $1 \mathrm{~mL}$ por vía intravaginal, una vez/día, durante 10 días consecutivos. Se observó el estado clínico general, así como la apariencia física de la vagina y el perineo por signos de flujo, eritema y edema. Al final del experimento se realizó la exéresis quirúrgica de la vagina de cada coneja y se evaluó macroscópicamente, buscando signos de irritación, sangrado o daño de la mucosa. Luego, se fijó en formol tamponado al $10 \%$ y posteriormente se tomó tres muestras representativas de las regiones cérvico-vaginal, media-vaginal, y urovaginal, para el estudio histopatológico. Principales medidas de resultados: Irritación vaginal. Resultados: El examen macroscópico de la vagina en el grupo control mostró aspecto congestivo leve en forma difusa, en todos los casos, y de aspecto similar en las muestras del grupo Croton lechleri al 1\%. En la formulación al 5\%, en dos casos se observó congestión petequial parcial leve. El estudio histopatológico reveló un puntaje promedio de irritación vaginal de 3,4 a 6 y 3,6 a 8 para las preparaciones al $1 \%$ y $5 \%$, respectivamente. Conclusión: En nuestras condiciones experimentales, las preparaciones de gel de $\mathrm{C}$. lechleri evaluadas fueron no irritantes para la mucosa vaginal y resultaron aceptables.
\end{abstract}

Palabras clave: Toxicidad; vagina; conejos; patología.

\begin{abstract}
Objective: To determine a Croton lechleri vaginal gel preparation toxicity in rabbits. Design: Experimental study. Setting: Alberto Guzman Barron Biochemistry and Nutrition Research Center, Clinical Research Institute, Institute of Pathology, Faculty of Medicine and Pharmacy Faculty, Universidad Nacional Mayor de San Marcos, Lima, Peru. Biological material: Croton lechleri resin and female white New Zealand rabbits. Methods: The animals were divided into three groups $(n=5)$ : I. Control, vehicle; II, C. lechleri $1 \%$, and III, C. lechleri $5 \%$; they received samples of $1 \mathrm{~mL}$ volume intravaginally 1 time/day for 10 consecutive days. The general clinical condition and physical appearance of the vagina and the perineum for signs of flow, erythema and edema were observed. At the end of the experiment surgical resection of the vagina of each rabbit was performed and signs of irritation, bleeding or mucosal damage were determined macroscopically. Specimens were placed in $10 \%$ buffered formalin and cervico-vaginal, mid-vaginal, and uro-vaginal samples underwent histopathology study. Main outcome measures: Vaginal irritation. Results: Gross examination of the vagina in both the control and the Croton lechleri $1 \%$ groups showed mild diffuse congestion. Two cases of Croton lechleri $5 \%$ formulation showed mild petechial congestion. Histopathology study revealed a vaginal irritation average score of respectively 3.4 to 6 , and 3.6 to 8 for the $1 \%$ and $5 \%$ preparations. Conclusions: In our experimental conditions, gel preparations of $C$. lechleri evaluated were acceptable and not irritating to the vaginal mucosa.
\end{abstract}

Key words: Toxicity; vagina; rabbits; pathology.

\section{INTRODUCCIÓN}

Las plantas medicinales, como el principal recurso en los sistemas médicos tradicionales, han sido utilizadas en la práctica médica durante miles de años y han hecho una gran contribución al mantenimiento de la salud humana. La mayoría de la población del mundo en los países en desarrollo todavía se basa en las plantas medicinales para satisfacer sus necesidades de salud. La Organización Mundial de la Salud (OMS) es plenamente consciente de la importancia de las plantas medicinales para la salud de muchas personas en todo el mundo, como se indica en una serie de resoluciones adoptadas por la Asamblea Mundial de la Salud y el Comité Regional para el Pacífico Occidental. Así pues, las plantas medicinales han sido reconocidas como un valioso recurso y de fácil acceso para la atención primaria de salud, y la OMS ha apoyado su uso seguro y eficaz ${ }^{(1)}$.

Croton lechleri Muell. Arg. es un árbol perteneciente a la familia Euphorbiaceae, de cuya corteza se extrae el látex viscoso de color rojo denominado sangre de grado o de drago, que es usado en la amazonia peruana y etnias nativas y que se ha difundido a todas las regiones, donde se vende comercialmente.

En la medicina tradicional ${ }^{(2)}$ se viene usando en forma indistinta la resina de Croton lechleri Muell. Arg. y Croton pa- lanostigma Klotzsch en el Perú, el Croton draconoides en Bolivia, como cicatrizante de cortes, heridas y en hemorragias; como antiinflamatorio en afecciones bucales y de la garganta; también en gastritis y úlceras ${ }^{(3,4)}$; es efectivo contra úlceras uterinas ${ }^{(5)}$ y se le usa para lavados vaginales para flujos e inflamaciones de los órganos genitales femeninos ${ }^{(2,6)}$.

Existen varios estudios fitoquímicos (7,8) y farmacológicos realizados en el extranjero y nacionales, en los que nuestro equipo ha contribuido con investigaciones que reconocen su valor protector en la mucosa gástrica de ratas, tanto de la resina como de su fracción alcaloidea denominada taspina ${ }^{(9-11)}$; asimismo, se 
ha profundizado en cierto grado sobre su probable mecanismo de acción (12-14). También, se ha realizado evaluaciones preclínicas que han validado sus propiedades cicatrizantes en la piel de animales de experimentación ${ }^{(3,15,16)}$ y se ha estudiado su potencial toxicológico ${ }^{(11,17-21)}$.

No se encuentra en la literatura científica estudios en el área ginecológica, por lo que es necesario conocer esta potencialidad lo más ampliamente posible, de acuerdo a las dosis usadas en animales de experimentación, siguiendo las buenas prácticas de laboratorio; por este motivo, realizamos el presente estudio, con la finalidad de evaluar el potencial efecto tóxico irritante vaginal de Croton lechleri en conejas.

\section{MÉTODOS}

Se realizó un estudio de tipo experimental, en el que se usó conejos hembras, vírgenes, de raza Nueva Zelanda, blancos, obtenidos del bioterio de la Facultad de Medicina Veterinaria de la UNMSM, de 2 a 2,2 kg de peso corporal. Los animales fueron mantenidos en el bioterio de la $\mathrm{Fa}$ cultad de Medicina de la Universidad Nacional Mayor de San Marcos; se les alojó en jaulas individuales y su alimentación fue con pellets de conejina obtenidos de Purina S.A., con agua ad libitum, en un ambiente con temperatura de $20^{\circ}$ a $23^{\circ} \mathrm{C}$, humedad relativa de 60 a $70 \%$, con ciclo de luz/oscuridad de 12/12 horas.

La resina de Croton lechleri fue obtenida de Agroselva S.A., proveniente de la Colonización Von Humboldt, Pucallpa, Departamento de Ucayali, Perú, extraída tres meses antes del experimento. El látex fue desecado en estufa a la temperatura de $40^{\circ} \mathrm{C}$ y el residuo obtenido se guardó en frasco oscuro a $4^{\circ} \mathrm{C}$ hasta su uso.

La formulación del gel vaginal se realizó en el laboratorio de Farmacotecnia de la Facultad de Farmacia y Bioquímica de la Universidad Nacional Mayor de San Marcos. El residuo desecado de Croton lechleri fue incorporado en polietilenglicol 400 en concentraciones de $1 \%$ y $5 \%$.

Para la evaluación de la toxicidad vaginal, después de una semana de aclimatización en el bioterio de la Facultad de Medicina de la UNMSM, las conejas fueron distribuidas aleatoriamente en los siguientes grupos de 5 animales cada uno:

I. Control, formulación del vehículo

II. Formulación de C. lechleri 1\%.

III. Formulación de C. lechleri 5\%.

La administración del vehículo (grupo I) y de la formulación de C. lechleri (grupos II y III), en volumen de $1 \mathrm{~mL}$, se realizó intravaginalmente con sonda metálica esterilizada de $6 \mathrm{~cm}$ de longitud, durante 10 días consecutivos, observándose y registrando para cada una de las conejas el estado clínico general, así como la apariencia física de la vagina y el perineo por signos de flujo, eritema y edema.

Al día siguiente de la última administración se efectuó la eutanasia de todos los animales mediante inyección de pentobarbital sódico en la vena marginal de la oreja, y posteriormente se los desangró. Se realizó la exéresis quirurgica de la vagina de cada coneja y se efectuó un corte longitudinal al canal vaginal, sujetándolo con alfileres en tecnoport e inmediatamente se evaluó macroscópicamente, buscando signos de irritación, sangrado o daño de la mucosa. Luego, se fijó en formol tamponado al 10\% y posteriormente se tomó tres muestras representativas de cada región vaginal: cervico-vaginal (CV), media-vaginal (MV) y uro-vaginal (UV); los cortes del órgano fueron incluidos en parafina y coloreados con hematoxilina-eosina.

De acuerdo a la severidad de la reacción, basado en el tipo de alteración histopatológica del tejido vaginal -ulceración epitelial, infiltración de leucocitos, edema y congestión vascular-, se asignó una puntuación a cada parte del órgano evaluada, según el sistema de clasificación de Eckstein y $\operatorname{col}^{(22,23)}$ (ausente $=0$, mínimo $=1$, leve $=2$, moderado $=3$, intenso $=4$ ), calculándose el promedio de puntajes en los grupos, que iba de 0 a 16. Este sistema de puntaje se correlaciona con el potencial de irritación en humanos, como sigue: puntaje de 0 a 8 es aceptable, de 9 a 10 potencial de irritación límite, y puntajes de 11 o mayores son indicativos de potencial irritativo significativo ${ }^{(24)}$.
Se calculó el índice de irritabilidad vaginal asignando una puntuación a cada parte del órgano evaluado, calculando el promedio de puntos. Al valor obtenido con C. lechleri al $1 \%$ y $5 \%$ se le restó el valor obenido por el control. Se consideró las categorías de respuestas en 0 ninguna, 1-4 mínima, 5-8 media, 9-11 moderada y $12-16$ severa $^{(24)}$.

Los valores de los puntajes de Eckstein fueron expresados como promedio \pm desviación estándar y las diferencias entre los grupos fueron determinadas mediante la prueba de Kruskal Wallis; se consideró diferencias significativas valores $p<0,05$.

\section{RESULTADOS}

Los animales toleraron bien el procedimiento durante el periodo de administración de diez días. No se apreció aspectos clínicos que mostraran signos de irritación vaginal en los animales tratados, excepto un animal del grupo Croton lechleri al 1\%, que presentó perineo y vulva congestionadas, al cual no se le administró el 5ำ día y despues cedió progresivamente.

El examen macroscópico de la vagina luego de su extracción, el grupo control mostró aspecto congestivo leve en forma difusa en todos los casos; el aspecto fue similar en las muestras del grupo Croton lechleri al $1 \%$, en algunos casos con predominio en el tercio externo (uro-vaginal). En el grupo de Croton lechleri al 5\%, en dos casos se observó congestión petequial parcial.

En la figura 1A se puede observar el epitelio cervico-vaginal de coneja administrada solo con vehículo, que hace un puntaje de 2, y en la figura 1B, de coneja que recibió Croton lechleri al 1\%, ninguna ulceración epitelial, e infiltración leucocitaria, edema y congestión vascular leves, que hacen un puntaje de 3 .

La figura 2 muestra el epitelio urovaginal de coneja del grupo Croton lechleri $1 \%$, también con un puntaje de 3 .

En cuanto al índice de irritabilidad vaginal calculado para la preparación del gel de C. lechleri al 1\%, alcanzó el valor máximo de 0,8 en las regiones media vaginal y urovaginal, mientras que para 


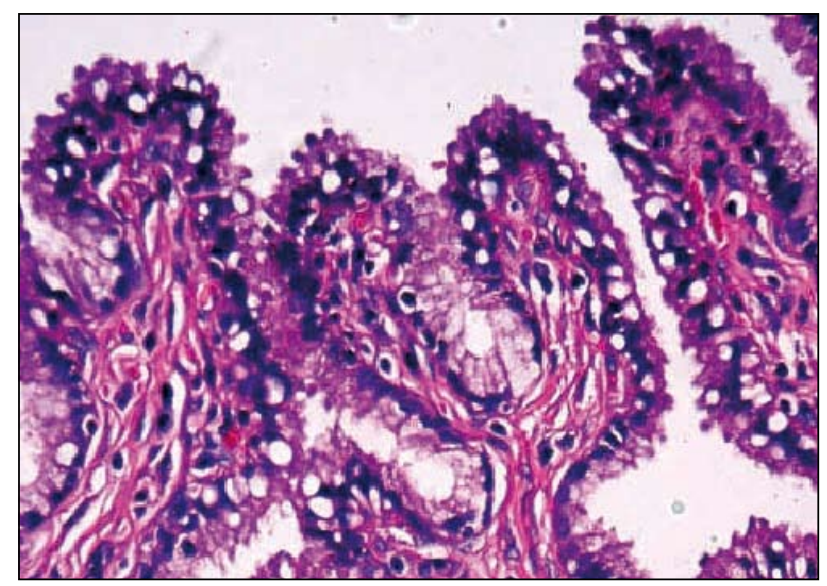

Figura 1A. Epitelio cérvico-vaginal de coneja del grupo control. Epitelio cilíndrico de aspecto glandular sin ulceración epitelial, leve infiltración leucocitaria, sin edema y leve congestión vascular. Coloración HE. 40x.

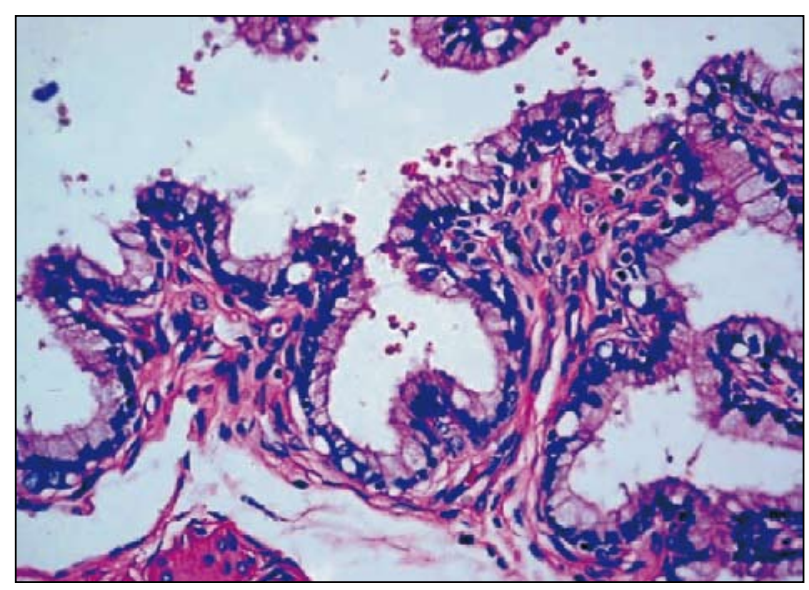

Figura 1B. Epitelio cérvico-vaginal de coneja del grupo Croton lechleri 1\%. Epitelio cilíndrico de aspecto glandular sin ulceración epitelial; infiltración leucocitaria, edema y congestión vascular leves. Coloración HE. 40x. la preparación al 5\%, el valor máximo fue 3 en la región uro-vaginal (figura 3). Estos valores recaen en la categoría de respuesta mínima.

\section{DISCUSIÓN}

Los estudios de toxicidad son fundamentales dentro del desarrollo de un nuevo fármaco y se extienden prácticamente a lo largo de todo el mismo. Se requiere las investigaciones toxicológicas para complementar la experiencia humana en la definición de la posible toxicidad a corto plazo, pero son especialmente importantes en la detección de la toxicidad que pueden producirse después de una exposición prolongada o años después que la exposición se ha interrumpido ${ }^{(1)}$.

Realizamos la investigación de irritación vaginal diferenciado en las zonas cérvico-vaginal $(\mathrm{CV})$, medio-vaginal (MV) y uro-vaginal (UV), debido a que histológicamente puede variar la sensibilidad de los irritantes, según sea el área de la mucosa vaginal; así, las zonas uro-vaginal y medio-vaginal están tapizadas por epitelio poliestratificado plano, mientras que la zona cérvico-vaginal, cercana al útero, está formada por epitelio cilíndrico ${ }^{(22)}$.

Es conocida la alta sensibilidad de la vagina de las conejas a irritabilidad de

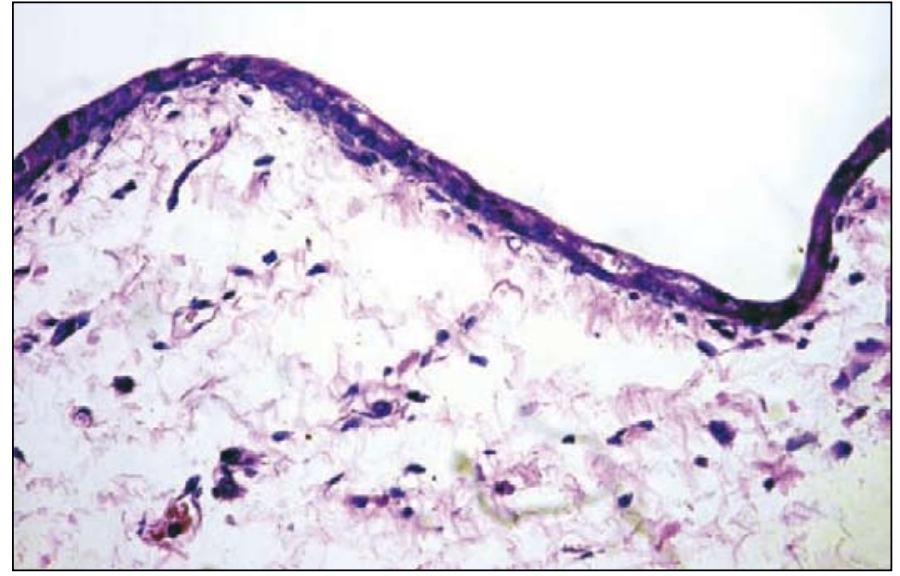

Figura 2. Epitelio uro-vaginal de coneja del grupo Croton lechleri $1 \%$. Epitelio poliestratificado plano sin ulceración epitelial; infiltrado leucocitario, edema y congestión vascular leves. Coloración HE. 40x. la mucosa. Durante la administración con cánula metálica y exposición a las sustancias durante 10 días, los resultados no mostraron irritación histopatológica significativa (figuras 1 y 2 ), ya que en el grupo Croton lechleri al $1 \%$ las puntaciones promedio por área de mucosa vaginal variaron de 0,4 a 2,6 y como promedios totales de 3,4 a 6 , muy similares al grupo en el que se administró el vehículo solo, en los cuales los promedios de irritación vaginal variaron de 0 a 1,6 y en promedio total alcanzaron de 3,2 a 5,2.

También, en el caso de una concentración mayor de Croton lechleri al 5\%, el promedio de puntuación varió de 0 a 3 y el promedio total de irritación vaginal de 3,6 a 8 .

Si consideramos lo propuesto por Eckstein y col ${ }^{(22,23)}$ sobre la irritación vaginal histopatológica -con una puntuación de 0 a 8 como aceptable, de 9 a 10 como irritación potencial límite (borderline) y por encima de 11 como potencial irritativo significativo-, entonces el grupo de Croton lechleri al 1\% -cuyo promedio fue 3,4 a 6-, está dentro del rango aceptable. Aún en el caso de una concentración alta de Croton lechleri al 5\%, la irritación llegó de 3,6 a 8, indicando una mayor irritación; en el caso de la región mediovaginal existió diferencia significativa $(p=0,04)$ con respecto al control, pero todavía en el rango aceptable, cercano al límite (tabla 1). 


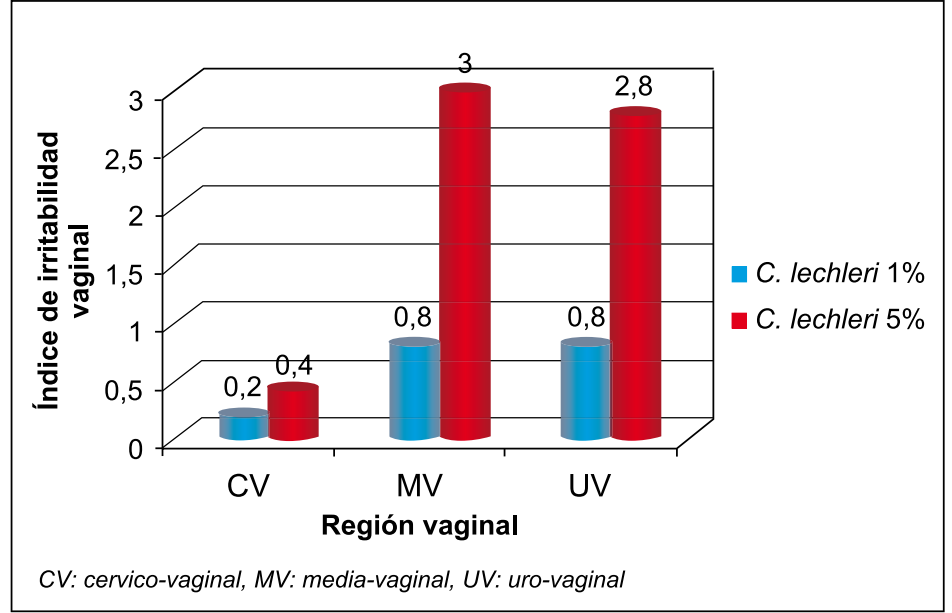

Figura 3. Índice de irritabilidad vaginal en conejas Nueva Zelanda tratadas con $\mathrm{C}$. lechleri durante 10 días.

Teniendo en cuenta el índice de irritación vaginal (figura 3), entonces, también los resultados de los valores recaen en la categoría de respuestas de índice mínimo de irritación.

Es pertinente mencionar que los resultados en las investigaciones iniciales con este modelo experimental mostraron variabilidad sustancial entre varios laboratorios ${ }^{(22)}$; pero, con su adecuada

estandarización, la Administración de Alimentos y Medicamentos de los Estados Unidos (US Food and Drug Administration) recomienda el uso de este modelo para evaluar el impacto tóxico local de antimicrobianos vaginales. El método de irritabilidad vaginal en conejas presenta varias ventajas sobre otros modelos animales, como permitir la administración de volúmenes relativamente grandes del

Tabla 1. Puntaje promedio de irritación vaginal para conejas blancas Nueva Zelanda tratadas con gel de C. lechleri por vía intravaginal durante 10 días consecutivos.

\begin{tabular}{lccc}
\hline $\begin{array}{l}\text { Componente vaginal } \\
\text { (Rango de puntaje) }\end{array}$ & $\begin{array}{c}\text { Control } \\
(\mathrm{n}=5)\end{array}$ & $\begin{array}{c}\text { C. lechleri } 1 \% \\
(\mathrm{n}=5)\end{array}$ & $\begin{array}{c}\text { C. lechleri } 5 \% \\
(\mathrm{n}=5)\end{array}$ \\
\hline $\begin{array}{l}\text { Cérvico-vaginal } \\
\text { Ulceración epitelial (0-4) }\end{array}$ & $0,0 \pm 0,0$ & $0,4 \pm 0,9$ & $0,0 \pm 0,0$ \\
Infiltración leucocitaria (0-4) & $1,4 \pm 0,5$ & $1,0 \pm 0,0$ & $1,4 \pm 0,5$ \\
Edema (0-4) & $0,8 \pm 0,4$ & $1,0 \pm 0,0$ & $1,0 \pm 0,0$ \\
Congestión vascular (0-4) & $1,0 \pm 0,0$ & $1,0 \pm 0,0$ & $1,2 \pm 0,4$ \\
Puntaje total (0-16) & 3,2 & 3,4 & 3,6 \\
Medio-vaginal & & & \\
Ulceración epitelial (0-4) & $1,2 \pm 0,4$ & $2,0 \pm 1,0$ & $2,0 \pm 1,0$ \\
Infiltración leucocitaria (0-4) & $1,0 \pm 0,0$ & $1,0 \pm 0,0$ & $1,6 \pm 0,5$ \\
Edema (0-4) & $1,2 \pm 0,8$ & $1,2 \pm 0,4$ & $1,6 \pm 0,5$ \\
Congestión vascular (0-4) & $1,0 \pm 0,0$ & $1,0 \pm 0,0$ & $2,2 \pm 1,3$ \\
Puntaje total (0-16) & 4,4 & 5,2 & $7,4 *$ \\
Uro-vaginal & & & $3,0 \pm 0,7$ \\
$\quad$ Ulceración epitelial (0-4) & $1,6 \pm 1,3$ & $2,6 \pm 0,5$ & $1,2 \pm 0,4$ \\
Infiltración leucocitaria (0-4) & $1,2 \pm 0,4$ & $1,2 \pm 0,4$ & $1,8 \pm 0,8$ \\
Edema (0-4) & $1,4 \pm 0,5$ & $1,2 \pm 0,4$ & $2,0 \pm 1,2$ \\
Congestión vascular (0-4) & $1,0 \pm 0,0$ & $1,0 \pm 0,0$ & 8 \\
Puntaje total (0-16) & 5,2 & 6 & \\
\hline
\end{tabular}

Valores expresados como promedio \pm Desviación estándar.

${ }^{*} p<0,05$ vs. Control, calculado con la prueba de Kruskal Wallis. compuesto $(1,0$ a $1,5 \mathrm{~mL})$ sin presentar derrame. La presencia de epitelio columnar, en parte de la vagina de la coneja, provee una alta sensibilidad a la prueba, confiriendo un razonable margen de seguridad del compuesto a probar para el uso en humanos. Adicionalmente, ha sido validado con investigaciones clínicas de fase I y seguido de amplia evaluación post-marketing ${ }^{(25)}$.

Actualmente, los investigadores están desarrollando pruebas más refinadas para evaluar la potencial irritación cérvicovaginal con el uso de antimicrobianos, en infecciones vaginales o uterinas, espermaticidas, anti-VIH, como la prueba mucosa de irritación vaginal ${ }^{(26)}$, histopatología e inmunofluorescencia con anticuerpos antineutrófilos ${ }^{(27)}$ y marcadores de inflamación solubles y celulares ${ }^{(25)}$.

Desde que existe correlación entre conejos y humanos respecto a la irritación vaginal con el empleo de diversos productos ${ }^{(23)}$, los resultados de este experimento apoyan el empleo del gel de Croton lechleri en una futura investigación clínica en humanos.

En conclusión, en nuestras condiciones experimentales, las preparaciones de gel de Croton lechleri en vehículo de polietilenglicol al $1 \%$ no fueron irritantes para la mucosa vaginal de conejas y resultaron aceptables.

\section{REFERENCIAS BIBLIOGRÁFICAS}

1. World Health Organization; Regional Office for the Western Pacific. Research Guidelines for Evaluating the Safety and Efficacy of Herbal Medicines. Manila: WHO; 1993.

2. Estrella E. Plantas medicinales amazónicas. Lima: Tratado de Cooperación Amazónica; 1995.

3. Meza E. Desarrollando nuestra diversidad biocultural. Sangre de Grado y el reto de su producción sustentable en el Perú. Lima: UNMSM; 1999.

4. Arellano P. El libro verde. Guía de recursos terapéuticos vegetales. Lima: Ministerio de Salud; 1992.

5. Barriga R. Plantas útiles de la Amazonía Peruana. Características, usos y posibilidades. Lima. Perú: Concytec; 1994.

6. Arévalo G. Las plantas medicinales y su beneficio en la salud. Shipibo, Conibo. Lima: AIDSEP; 1994.

7. Persinos G, Farnsworth N, Blomster R, Blake D. Studies on South American plants I. Taspine 
isolation and anti-inflammatory activity. Lloydia. 1974:37:644C.

8. Cai F, Evans M, Roberts J, Phillipson M, Zenk M, Glebas Y. Phytochemistry. 1991;30(6):2033-40.

9. Ayala S, Díaz D, Palomino M, Armas S, Paz J. Efecto protector del Croton palanostigma y Aloe vera frente a la injuria aguda de mucosa gástrica inducida por etanol en ratas. An Fac med. 1999;60(1):22-9.

10. Ayala S, Jurupe H, Díaz D, Lock O, Vega M, Luque J, et al. Efecto protector del látex desecado y fracción alcaloidea de Croton palanostigma frente a injuria de mucosa gástrica inducida por etanol en ratas. An Fac med. 2001;62(4):317-24.

11. Miller M, Mac W, Zhang X, Sandoval M. Treatment of gastric ulcers and diarrhea with the Amazonian herbal medicine sangre de grado. Am J Physiol Gastrointest Liver Physiol. 2000;279:G192G200.

12. Sandoval M, Ayala S, Oré R, Arroyo J. Inducción de la formación de moco gástrico por sangre de grado Croton palanostigma. An Fac med. 2002;63(4):251-6.

13. Sandoval M, Ayala S, Oré R, Ricra V, Durand J, Ugarte G. Estimulación de la actividad péptica del jugo gástrico inducida por látex de Croton palanostigma (sangre de grado). An Fac med. 2003;64 Supl 1:24.

14. Sandoval M, Ayala S, Oré R, Ricra V, Ugarte G. Incremento del moco gástrico y reducción de grupos sulfidrilo no proteícos por estimulación con sangre de grado de Croton palanostigma. An Fac med. 2004;65 Supl 1:21.

15. Vaisberg A, Milla M, Planas M, Córdova J, Augusti $E$, Ferreira $R$, et al. Taspine is cicatrizant principle in sangre de grado extracted from Croton Lechleri. Planta Med. 1994;60(6):541-5.
16. Porras B, Lewis W, Roman J, Simchowitz L, Mustoe T. Enhancement of wound healing by the alkaloid taspine defining mechanism of action. Proc Soc Exp Biol Med. 1993;203(1):18-25.

17. Itokawa H, Ichihara Y, Mchizuki M, Enomori T, Moria $\mathrm{H}$, Shirota $\mathrm{O}$, et al. A cytotoxic substance from sangre de grado. Chem Pharm Bull (Tokio). 1991;39(4):1041-2.

18. Rossi D, Bruni R, Bianchi N, Chiarabelli C, Gambari $\mathrm{R}$, Medici A, et al. Evaluation of the mutagenic, antimutagenic and antiproliferative potential of Croton lechleri (Muell Arg) latex. Phytomedicine. 2003;10:139-44.

19. Shen Y, Bryngelsson C, Pero R. Enhanced DNA repair, immune function and reduced toxicity of C-MED-100. J Ethnopharmacol. 2000;69:11526.

20.Sandoval M, Ayala S, Oré M, Valdiviezo L, Loli R, Rivera V, et al. Evaluación de la toxicidad hepática y renal subaguda del látex de Croton palagnostigma (sangre de grado) en animales de experimentación. An Fac med. 2005;66(2):11926.

21. Ayala S. Toxicología aguda el Croton lechleri (sangre de grado) en especies de laboratorio. Tesis de Maestría de Medicina. Lima: Universidad San Martín de Porres; 2005

22. Eckstein P, Tackson M, Millman N, Sobrero A. Comparison of vaginal tolerance test of spermicidal preparation in rabbits and monkeys. J Reprod Fertil. 1996;20:85-93.

23. D'Cruz OJ, Samuel P, Uckun FM. Conceival, a novel noncontraceptive vaginal vehicle for lipophilic micobicides. AAPS PharmSciTech. 2005;6(1):E5664.

24. Agüero F, Bulnes C, Durand R, García L. Ensayo de irritabilidad vaginal a un producto natural, obtenido a partir de Rhizophora mangle L. destinado al tratamiento de infecciones uterinas. REDVET [Internet]. 2005[citado el 25 de agosto de 2009]:VI(6):[aprox. 8 p.]. Disponible en: http://www.veterinaria.org/revistas/redvet/ n060605/060516.pdf

25. Doncel G, Chandra N, Fichorova R. Preclinical assessment of the proimflammatory potential of microbicide candidates. JAIDS. 2004:37:S17480.

26. Dhondt M, Adriaens E, Roey J. Remon J. The evaluation of the local tolerance of vaginal formulations containing dapivirine using the Slug Mucosal Irritation test and the rabbit vaginal irritation test. Eur J Pharm Biopharm. 2005;60(3):419-25.

27. D'Cruz 0, Samuel P, Waurzyniak B, Uckun F. Development and evaluation of a thermoreversible ovule formulation of stampidine, a novel nonspermicidal broad-spectrum anti-HIV microbicide. Biol Reprod. 2003;69:1843-51.

Fuente de financiamiento: Facultad de Medicina de la UNMSM, $5^{\circ}$ Concurso de Proyectos de Investigación.

Manuscrito recibido el 17 de febrero de 2010 yaceptado para publicación el 28 de mayo de 2010.

\section{Correspondencia:}

Salomón Ayala Pío

Facultad de Medicina, UNMSM.

Av. Grau 755.

Lima 1, Perú.

Correo-e: sayalap1@hotmail.com 